\title{
GESTIÓN ADMINISTRATIVA Y CONOCIMIENTO DE LAS TIC EN DOCENTES DE EDUCACIÓN PRIMARIA DE LAS INSTITUCIONES EDUCATIVAS INNOVA SCHOOLS DE SAN JUAN DE LURIGANCHO Y ATE
}

\section{Administrative management and knowledge of ICT in primary education teachers at educational institutions Innova Schools of San Juan de Lurigancho and Ate}

\author{
Pedro José Farroñay Díaz ${ }^{1}$ \\ I.E. Teresa Gonzáles de Fanning, Perú \\ María del Carmen Emilia Ancaya Martínez² \\ Universidad Peruana Cayetano Heredia, Perú
}

\section{RESUMEN}

El objetivo de esta investigación fue determinar la relación entre la gestión administrativa y el conocimiento de las TIC, según la percepción de los docentes del nivel primaria de la Institución Educativa Innova Schools en las Sedes de San Juan de Lurigancho y Ate en el año 2013. Investigación básica que se enmarca dentro del enfoque cuantitativo, con un alcance descriptivo y diseño no experimental, transversal correlacional. Se realizó con una muestra censal de 155 docentes del nivel Primaria. Se utilizó la técnica de la encuesta, con dos cuestionarios que midieron las variables gestión administrativa y conocimiento de las TIC. Ambos instrumentos se validaron con la opinión de expertos y su confiabilidad se verificó mediante el coeficiente de consistencia interna alfa de Cronbach. Los resultados demostraron que existe una relación baja $\left(\right.$ rho $\left.=.335^{* *}\right)$, pero directa y significativa entre la gestión administrativa y el conocimiento de las TIC en docentes del nivel Primaria de la mencionada institución educativa. Por lo que se concluye afirmando que a mejor gestión administrativa, mejor será el conocimiento de las TIC en los docentes.

Palabras Clave: Gestión administrativa, gestión tecnológica, gestión financiera, conocimiento de las TIC.

\section{Abstract}

The objective of this research was to determine the relationship between administrative management and the knowledge of ICT, as perceived by primary level teachers in the educational institution Innova Schools at the San Juan de Lurigancho and Ate buildings in the year 2013. This is a basic research, which fits within the quantitative approach with a descriptive scope and a non-experimen-

1 Magister en educación: Docente del Área de Educación para el Trabajo, especialista en Tecnologías de la Información y Comunicación (TIC), Docente del Aula de Innovación Pedagógica del CEBA Teresa González de Fanning, Sub Director de Gestión de Materiales Educativos del Instituto Nacional de Defensa Civil, publicaciones de textos sobre Gestión Reactiva del Riesgo, expositor sobre Tecnologías de la Información y Comunicación (TIC). Email: josefarronay@outlook.com

2 Magister en educación: Maestría en Administración de la Educación, Licenciada en Matemáticas Aplicadas de la Universidad Peruana Cayetano Heredia. Especialista en Ing. de Sistemas en la Universidad de Lima. Docencia en Estadística y Matemáticas, experiencia en trabajos multidisciplinarios en la UPCH. 
Gestión administrativa y conocimiento de las TIC en docentes de educación primaria de las instituciones educativas Innova Schools de San Juan de Lurigancho y Ate

tal, crossectional and correlational design. It was a total population sampling including 155 teachers from primary level of the educational institution Innova Schools at the San Juan de Lurigancho and Ate buildings. The survey research technique was used, with two questionnaires that measured the variables: administrative management and the knowledge of ICT. Both instruments were validated by experts opinion and the reliability was verified by the Cronbach's alpha coefficient. The results showed that there is a low ratio $\left(\mathrm{rho}=.335^{* *}\right)$, but direct and significant relationship between the administrative management and the knowledge of ICT in primary level teachers of the educational institution. To conclude, we state that the better the administrative management, the better the knowledge of ICT in teachers will be.

Keywords: Administrative management, technology management, financial management, knowledge of ICT.

\section{INTRODUCCIÓN}

Existen diversas investigaciones relacionado al uso de las tecnologías de la información y comunicación (TIC) para gestionar administrativamente a nivel educativo. En Europa, Selwood (s.f) refiere en su estudio que las actitudes de los docentes hacia el valor de las TIC y su uso para la administración y gestión son positivas para el apoyo en sus funciones pedagógicas y administrativas, sin embargo al analizar los resultados muestran un bajo nivel de utilización de las TIC, enfatizando que se debe a la falta de formación en estos medios, la disponibilidad de tiempo y recursos tecnológicos idóneos.

En Latinoamérica, en Chile, Lagos (2011) analizó las percepciones respecto al uso de herramientas TIC en el aula y concluyo que los docentes son un poco reacios al uso y utilidad de estos materiales en la enseńanza, siendo necesario comprometerlos en un proyecto que responda a las necesidades de la comunidad y así evitar el rechazo a posibles intervenciones futuras. En México, Farjart (2009) estudió las percepciones y actitudes de los profesores sobre el uso de las tecnologías de la información y comunicación en el proceso aprendizaje-enseñanza y llegó a la conclusión de que el uso de estas es positivo, por lo que recomendó su uso para generar un aprendizaje acorde con estos tiempos. En este mismo país Silva, Cruz y Hernández (2013) estudiaron la importancia de los Sistema de Gestión Digital en el mejoramiento de los procesos administrativos de instituciones de Educación Superior y llegaron a la conclusión de que es necesario implementar sistemas de gestión digital funcionales que permitan optimizar recursos en las instituciones educativas tomar decisiones estratégicas y pertinentes para la sociedad, por su parte Perera (2012) analizó las opiniones de alumnos, docentes y autoridades escolares acerca de las TIC y llegó a la conclusión de que aparentemente estas se están utilizando, pero sin que haya diferencias significativas en el aprendizaje. En Colombia, Mejía (2011) estudió las percepciones, uso y apropiación de TIC en los docentes llegando a la conclusión de que si bien los docentes consideran que las TIC son herramientas que ayudan a mejorar los procesos de enseńanza y aprendizaje, en los últimos años las usan dejando de lado la importancia didáctica o pedagógica que ésta puede tener. El Perú, no está exento de este análisis, Joo (2004) realiza una propuesta de gestión pedagógica y administrativa de las TIC, para construir espacios que generen conocimiento en el Colegio Champagnat, concluyó que en el campo administrativo del sector educación las nuevas tecnologías de la información agilizan los procesos administrativos y son el soporte de la actividad docente. El tratar de asociar la gestión administrativa y el conocimiento de las TIC según la percepción de los docentes, es una tarea compleja, dada la situación diversa que presenta una escuela que cuenta con recursos limitados y donde, además, los docentes tienen rechazo o desinterés por las innovaciones tecnológicas, en especial por las referidas al uso de las herramientas TIC y las plataformas educativas que ofrece la Institución Educativa Innova Schools. 
Desde este ámbito creemos que era necesario ahondar más en el tema, por lo que se partió de la pregunta ¿Cuál es la relación entre la gestión administrativa y el conocimiento de las TIC, según la percepción de los docentes del nivel primaria de la Institución Educativa Innova Schools en las Sedes de San Juan de Lurigancho y Ate en el año 2013?, formulándose como objetivo general: determinar la relación entre la gestión administrativa y el conocimiento de las TIC, según la percepción de los docentes del nivel primaria, y como objetivos específicos: (i) Determinar la relación entre la gestión de recursos tecnológicos y el conocimiento de las TIC, según la percepción de los docentes del nivel primaria; y (ii) Determinar la relación entre la gestión de recursos financieros y el conocimiento de las TIC, según la percepción de los docentes del nivel primaria.

\section{Gestión ADMinistrativa}

Antes de dar una definición de gestión administrativa, es relevante definir de manera individual los términos gestión y administración. Según Aguerrondo (1990) refiere que gestión, es toda medida que supone un componente político, en tanto tiende a concretar una intencionalidad. Cuando el ámbito de aplicación es la institución escolar, el interés de la acción es obtener determinados resultados pedagógicos a través de la actividad educativa escolar, llevada a cabo por cada comunidad educativa particular (p. 60). Indicando además que todos los miembros de la institución escolar implementan diariamente decisiones de política educativa cuando organizan equipos de trabajo en el aula y en la institución, cuando toman medidas administrativas y de gestión del establecimiento, cuando definen los mecanismos de inscripción de los estudiantes, las modalidades de evaluación de sus aprendizajes, etcétera. En cuanto a la definición de administración, Henry Fayol (considerado el verdadero padre de la administración moderna), afirma que "administrar es prever, organizar, mandar, coordinar y controlar" (Reyes, 2010, p. 16). En ese sentido, la administración debe cumplir la función de lograr que las cosas se realicen por medio de otros, $\mathrm{u}$ obtener resultados a través de otros. La administración es un fenómeno universal en el mundo moderno. Cada organización debe alcanzar objetivos en un ambiente de competencia acérrima, debe tomar decisiones, coordinar múltiples actividades, dirigir personas, evaluar el desempeño en base a objetivos determinados, conseguir y asignar recursos, etc. Chiavenato (1990, p. 42), aseverando además que la administración debe cumplir ciertos procesos que permitan alcanzar y lograr sus objetivos.

Desde esta perspectiva al hacer referencia a Gestión administrativa. Este concepto aúna las nociones de gestión y administración. En opinión de Antúnez (1993), la dimensión administrativa de la gestión, es el conjunto de acciones que se realizan a fin de movilizar recursos (personas, tiempo, dinero, materiales, etcétera.) para la consecución de los objetivos de la institución. Administrar la educación, en tanto política pública y de interés general, se liga a la acción de gobernar, y requiere, por tanto, de un aparato que haga posible la planificación, la prevención de suministros, el procesamiento de la información para la toma de decisiones y la implementación de acciones.

La gestión administrativa es la optimización en la ejecución de los procesos con el fin de incrementar la cantidad y eficiencia en la gestión de los servicios (Ministerio de Educación de Guatemala, 2004, p. 17). Algunas acciones concretas serán la administración del personal, desde el punto de vista laboral, asignación de funciones y evaluación de su desempeño; el mantenimiento y conservación de los bienes muebles e inmuebles; organización de la información y aspectos documentarios de la institución; elaboración de presupuestos y todo el manejo contable-financiero (UNESCO, 2011, p. 36).

Gestión administrativa educativa. Hablar de administración educativa es referirse a un sistema o proceso para organizar, dirigir, estructurar y dar vida la implementación de un servicio educativo en un medio social que lo requiere, con la finalidad de impartir un servicio de enseñanza-aprendizaje que permita a los estudiantes aprender de acuerdo con sus necesidades cognitivas, de aplicación, personales y sociales (Martínez, 2012, p. 32). Según Martínez, la Administración Educa- 
Gestión administrativa y conocimiento de las TIC en docentes de educación primaria de las instituciones educativas Innova Schools de San Juan de Lurigancho y Ate

tiva debe observar la organización, la dirección y el buen manejo de la misma, desde un adecuado uso de los recursos que componen a cualquier organización con enfoque financiero y de servicios. Incorporar los recursos tecnológicos de cada establecimiento en actividades pedagógico-didácticas que se integren al Proyecto Educativo Institucional implica apreciar críticamente el valor de estos para la articulación de la educación y el trabajo (Barbeito, 2004, p. 8). Este mismo autor hace énfasis en indicar que los docentes y directivos participantes deberán tener conocimiento básicos de cómo operan las herramientas informáticas. De ahí que la integración de recursos tecnológicos al currículo escolar debe ser un proceso gradual, vinculado a varios factores: Los recursos tecnológicos propiamente dichos; la disponibilidad y correcta utilización de los contenidos digitales apropiados; la propuesta pedagógica; la competencia tecnológica de los educadores y el apoyo administrativo y técnico que ofrece la institución educativa.

Gestión de recursos tecnológicos. La gestión tecnológica, dentro de un marco administrativo eficaz, permite una apropiada interacción entre la tecnología, el recurso humano y el conocimiento generado y asimilado, lo que conlleva a aumentos en la calidad de los bienes o servicios ofrecidos, en la productividad y en la competitividad.

Cabe puntualizar que la generación, la asimilación y el uso efectivo de la tecnología mediante la gestión tecnológica, incluye también la utilización eficiente de los recursos empleados por la empresa, al igual que la minimización del impacto que tiene la actividad productiva sobre el ambiente. Castellanos (2007, p. 19) analiza la importancia de la gestión en el desarrollo tecnológico para lograr la competitividad de las organizaciones. Para ello, en primer lugar se presenta el contexto general sobre el desarrollo tecnológico, la conceptualización de tecnología y su relación con la ciencia y la gestión, por su parte Morelos (2011), indica que los recursos tecnológicos más utilizados en el ámbito educativo son el computador, el tablero electrónico, la televisión, los videos/CDs y el proyector. Para Krishnaveni \& Meenak (2010) refieren que la integración de las TIC ayuda a reducir la complejidad en la gestión educativa, ya que al integrar esta permite mejorar la eficiencia de acti- vidades, como en la administracion de datos del estudiante, gestionar las nominas, contabilidad financiera, sistemas de bibliotecas, administrar las actividades del día a dia de la institución.

Gestión de recursos financieros. Por gestión de recursos financieros se entiende a un conjunto de procesos coordinados e interdependientes encaminados a planificar, organizar, controlar y evaluar los recursos económico-financieros disponibles en la organización, de cara a garantizar de la mejor manera posible la consecución de unos objetivos sociales fijados previamente y coherentes con su misión (Gavilán, 2008, p. 20). Se habla de un conjunto de procesos, es decir, una serie de pasos y acciones sucesivas e interrelacionadas dirigidas a alcanzar eficientemente unos objetivos o resultados sociales finales.

Los gastos en las escuelas son diversos. Entre los rubros más importantes están:

- Pago de servicios básicos y de comunicación (luz, agua, gas, teléfono e Internet).

- Mejora de infraestructura (construcción de aulas, reparación de paredes, protecciones para ventanas, entre otros).

- Adquisición y reparación de mobiliario (estantes, libreros, mesas y sillas).

- Compra de equipo (grabadoras, computadoras y televisores).

- Pago de personal de apoyo a la docencia (maestro de educación física, artística, computación e inglés).

Se considera que los gastos de operación de las escuelas involucra la atención de las necesidades relacionadas con servicios, recursos materiales e infraestructura, como el mantenimiento y la mejora física de los planteles, la adquisición y la reparación de mobiliario, la compra de equipos y tecnología de la información y la comunicación (Saavedra, 2002, p. 39).

\section{Conocimiento de las TIC}

Tecnologías de la Información y la comunicación (TIC). Según Marqués (2008, p. 38), las TIC son un instrumento indispensable para las instituciones educativas; que le permiten realizar 
numerosas funciones. Sirven como fuente de información multimedia hipermedial, canal de comunicación para el trabajo colaborativo, medio de expresión para la creación, instrumento cognitivo y para procesar la información, medio didáctico y herramienta para la gestión.

Dominio de las TIC. El uso de las TIC en la escuela busca desarrollar en los estudiantes capacidades y actitudes que les permitan utilizar y aprovecharlas adecuadamente dentro de un marco ético, potenciando el aprendizaje autónomo a lo largo de la vida. Se requiere formarlos en el dominio de las tecnologías de la información y comunicación digital (Internet), con capacidad para desempeńarse de forma competente en el uso de los diversos programas para la recopilación análisis, interpretación y uso de información pertinente para la solución de problemas y toma de decisiones de manera eficaz. La escuela ofrece una formación que desarrolle el juicio crítico y el pensamiento estratégico y reflexivo de los estudiantes. Con ellos se pretende que sepan seleccionar las fuentes de información y herramientas pertinentes de soporte a los proyectos que emprendan, así como identificar nuevas oportunidades de inclusión a través de comunidades virtuales. Igualmente, la escuela busca adaptarse a los efectos que este lenguaje digital tiene en las maneras de aprender y comunicarse de los estudiantes (Ministerio de Educación, 2009, p. 30).

Las TIC en la enseñanza. En el ámbito educativo, las TIC se convierten en aliadas perfectas para la innovación educativa; facilitan la colaboración entre personas que comparten intereses y habilidades, sin importar el lugar donde se encuentren; su uso representa una transformación del significado de la educación, que ya no se limita al espacio de un aula de clase, sino que se abre al poder de la red, el nuevo aliado en la tarea educativa (Ministerio de Educación Nacional, 2013, p. 20). Para Cabero (2007, p. 43), las nuevas tecnologías de la información y la comunicación se utilizan para referirse a una serie de medios nuevos, como los hipertextos, los multimedios, Internet, la realidad virtual o la televisión por satélite. Este mismo autor, señala que dichas tecnologías tienen un carácter de interactividad en torno a las teleco- municaciones, la informática, los audiovisuales y su hibridación, como son los multimedia. Como señala Castell (2001, p. 36), la sociedad red está construida en base a redes de información a partir de las tecnologías de la información. Internet no es solo una tecnología; es el medio de comunicación que favorece la organización social; es el corazón de un nuevo paradigma socio-técnico que constituye la base material de la vida del hombre y sus formas de relacionarse, trabajar y comunicarse. Asimismo, Burbules (2008, p. 34) señala que los estudiantes cuentan con la oportunidad de ampliar su experiencia de aprendizaje al utilizar las TIC como herramientas para construir su aprendizaje. Estas herramientas permiten que el aula tradicional se convierta en un nuevo espacio, con actividades innovadoras de carácter colaborativo y oportunidad para la creatividad; los estudiantes aprenden al mismo tiempo que se divierten. Por otro lado, los docentes pueden estrechar lazos con otros profesores que se hallan en lugares distantes; de no ser por las TIC, nunca hubieran tenido la oportunidad de colaborar entre sí.

Herramientas informáticas. La información será el conjunto de datos y los resultados que proporciona la máquina (Nacho, 1999, p. 35). La ciencia informática estudia el procesamiento automático de la información, aunque la necesidad de razonar sobre este tipo de procesos existe desde tiempo atrás. La consolidación de la informática como ciencia sólo se produce con el desarrollo de los computadores, a partir de los años cuarenta. Por lo tanto, se trata de una ciencia muy joven, pero que ha evolucionado a gran velocidad. La piedra maestra sobre la cual se ha podido desarrollar la informática es el computador, herramienta de gran eficacia en muy diversos trabajos $y$, en particular, en aquéllos que manejan un gran volumen de datos u operaciones (Pareja, 1994, p. 17). Dentro de estas herramientas es importante mencionar al computador, que es una máquina electrónica que procesa información siguiendo las instrucciones de un programa registrado. Para comunicarse con el exterior, el ordenador dispone de unos medios de entrada a través de los que recibe la información y unos medios de salida, por donde la envía. Tiene dispositivos que le permiten almacenar la información (datos, resultados 
Gestión administrativa y conocimiento de las TIC en docentes de educación primaria de las instituciones educativas Innova Schools de San Juan de Lurigancho y Ate

y el propio programa) y procesarla siguiendo las instrucciones del programa. La información que se procesa en el computador (programas, datos $y$ resultados) se expresa en forma digital binaria, combinando ceros y unos. En consecuencia, tanto los programas como los datos y resultados deben codificarse en este formato para poder ser procesados (Pareja, 1994, p. 18).

La informática y la educación. Para utilizar la informática en el aula, el profesorado tendrá en cuenta una serie de aspectos, basados en la participación e interés del alumno hacia el proceso de aprendizaje-enseñanza y buscará las metodologías que mejor se adapten a su ritmo educativo, mediante el diseńo de modelos independientes y autónomos. La información y los contenidos que se transmiten a través del equipo informático tendrán una presentación adaptada a las necesidades y características personales de cada estudiante, al mismo tiempo que se favorecerá la interacción entre este y el uso de los medios informáticos (Salinas, 2004, p. 22). La informática proporciona nuevas propuestas y modelos de aprendizaje desarrollados principalmente dentro de la inteligencia artificial (Romero, 1993, p. 17).

Medios informáticos. Se caracterizan por estar configurados en un software y articulados por el computador y presentan las siguientes cualidades: Gran flexibilidad por su estructura no lineal, alta interactividad, aprendizaje autodirigido y la persona construye su conocimiento de forma individual o grupal.

El computador ofrece una serie de presentaciones didácticas: Microsoft Office (Word, Power Point, Excel, entre otros), hipertexto, multimedia, video interactivo, Macromedia Flash y otras muchas más, que brindan al usuario la posibilidad de realizar eficientemente una tarea específica.

\section{Herramientas telemáticas}

Gracias a las TIC y mediante el uso de medios informáticos es posible transmitir conocimientos vía telemática, es decir, a través de las redes. Una red consiste en la conexión de dos o más computadoras conectadas por un cable u otro dispositi- vo que permita intercambiar datos; estos cables están a su vez conectados a las tarjetas de red, que son dispositivos electrónicos que se instalan en las computadoras.

Internet. Es la red mundial de redes de computadores, que permite a éstos intercomunicarse para compartir información y servicios a lo largo y ancho del mundo. Esta red no es propiedad de nadie, sino un conjunto de redes interconectadas que son públicas e internacionales, dedicadas a la investigación, a la información al entretenimiento y al comercio. Desde su inicio como una red de investigación y de uso militar, ha pasado a convertirse en la auténtica precursora de las superautopistas de la información por donde se transmiten imágenes, tanto fijas (dibujos y fotografías) como en movimiento (videos, imágenes animadas), e incluso imágenes en tres dimensiones, sonidos, voz y una gran cantidad de datos.

Algunas de las características que han determinado la creciente popularidad de este sistema:

- Realización de la mayoría de los procedimientos mediante documentos de hipertexto, que permiten navegar intuitivamente por las distintas fuentes de información mediante los hiperenlaces.

- Uso de técnicas multimedia; un documento de hipertexto puede combinar textos, imágenes, vídeo, sonido.

- Los progresivos avances en los programas diseñados para navegar por Internet, que facilitan cada vez más el uso de los recursos, tales como Netscape Navigator o IExplorer.

- La interactividad, permite recibir y enviar información.

Intranet. Es una red interna de computadores al servicio de una institución o una empresa. No es una red pública como el internet. A ella solo puede tener acceso los integrantes de la propietaria de la red. Se trasmite información de interés para la entidad.

Correo electrónico. También conocido como email, es la herramienta más utilizada para enviar y recibir mensajes entre los usuarios de la red en cualquier lugar del mundo. El correo electrónico es una de las aplicaciones más utilizadas en In- 
ternet; su facilidad de uso, su aspecto práctico y su inmediatez ha popularizado las trasmisiones telemáticas en numerosas actividades científicas y económicas.

Teleconferencia vía internet. Con los actuales avances tecnológicos de las redes es posible hacer teleconferencias por medio del computador, que involucran la imagen y el sonido, para trasmitir información a uno o más usuarios. La teleconferencia aporta al instructor un recurso para ilustrar conceptos, presentar diversas fuentes de información, propiciar entornos con gran capacidad de motivación y generar discusiones.

El chat. Es una herramienta que permite la conversación entre dos o más personas en tiempo real (independientemente donde se encuentren). Su funcionamiento depende de un programa de aplicación denominado Internet Relay Chat (IRC), creado en 1988 por Jarkko Oikarinem con el objetivo de introducir mejoras en un programa llamado Talk (para establecer contacto entre dos ordenadores). El chat permite la intercomunicación de varios usuarios, observando las personas que participan y los mensajes que están escribiendo. Para chatear, el usuario debe conectarse a un servidor, a través del que accede a cualquiera de los canales o salas disponibles para ello, que reciben el nombre de la temática tratada en ellos (Rubio, 2010, p. 36).

Foros. En los foros deben guardarse unas normas de cortesía entre las que cabe citar no escribir en mayúscula, respetar opiniones no imponiendo la propia, atender a la correcta redacción y expresión evitando las faltas de ortografía (Rubio, 2010, p. 62).

Video conferencia. Permite al usuario ver a la vez que se está hablando con él. También se le llama videoconferencia de escritorio o Desktop videoconferencia. Es como un videoteléfono y resulta muy apropiado para seguir una conferencia o clase a distancia (Clares, 2000, p. 196). El desarrollo de las telecomunicaciones ha ampliado las posibilidades de comunicación entre los usuarios y ha dado lugar a la aparición de una amplia gama de servicios que tienen su proyección también en el campo de la formación. Esta evolución de las telecomunicaciones reduce las distancias, de tal forma que actualmente es posible hablar de grupos de formación compuestos por personas separadas geográficamente. La videoconferencia se caracteriza por presentar diversas fuentes de información y proporciona entornos con gran capacidad de motivación.

\section{Conocimiento de InTERnet}

Internet es una red mundial de redes de ordenadores, que permite a éstos comunicarse en forma directa y transparente, compartiendo información y servicios a lo largo de la mayor parte del mundo (Altuna, 2010, p. 33).

Internet en la clase. Cada día, más escuelas están conectadas a Internet; al ser está una fuente inagotable de información y datos de primera mano. Como red originariamente científica, en Internet puede encontrarse gran cantidad de información útil para la clase, desde imágenes de satélites meteorológicos recién tomadas a documentos históricos pasando por conjuntos de datos sobre ecología o el último paper no publicado todavía sobre algún tema específico, un bagaje de información que se actualiza en segundos, lo cual permite nutrir el conocimiento de manera permanente.

Estudiantes de lugares físicamente distantes utilizan la red como medio de comunicación para realizar proyectos colectivos, recoger e intercambiar datos sobre aspectos diferentes de su medio ambiente o estudiar las diferencias y semejanzas culturales entre comunidades de diferentes países. En un revival sorprendente, una especie de "Freinet electrónico" se apodera de las aulas conectadas a la red: los estudiantes se enzarzan en correspondencias escolares electrónicas y multimediáticas con compañeros de la otra parte del mundo (Adell, 1993, p. 54).

Para utilizar los recursos de Internet, debe fomentarse en los usuarios una actitud crítica hacia la información circulante. El volumen y la diversa confiabilidad de los productores de la información que hay en Internet ponen en primer plano la cuestión de la capacidad del usuario para dife- 
Gestión administrativa y conocimiento de las TIC en docentes de educación primaria de las instituciones educativas Innova Schools de San Juan de Lurigancho y Ate

renciar la información relevante y veraz de la que no lo es. Sólo la construcción de criterios de selección, el desarrollo de destrezas de alto orden y la adquisición de valores, permitirá a los usuarios seleccionar la mejor información. Sin duda, la falta de conocimiento sobre una temática, complica la búsqueda de nueva información hasta transformarla en un laberinto de difícil salida (Sánchez, 1995, p. 11).

\section{Materiales y Métodos}

Las variables estudiadas son el progrma de enseñanza de las TIC (X) y competencias tecnológicas en docentes $(\mathrm{Y})$. El trabajo realizado analizó el efecto del programa en el desarrollo de las competencias tecnológicas de los docentes de los Centros de Educación Básica Alternativa (CEBA). En las secciones siguientes se detalla el método de trabajo utilizado para verificar el efecto de la variable $\mathrm{X}$ en la variable $\mathrm{Y}$.

\section{Participantes}

La población en estudio estuvo conformada por 155 docentes de la Institución Educativa Innova Schools de las Sedes de San Juan de Lurigancho y Ate. La muestra, de tipo censal, incluyó al 100\% de docentes de las referidas instituciones educativas. No fue necesario fijar un tamaño muestral debido a la poca cantidad de docentes que laboran en las referidas sedes.

\section{Tipo y Diseńo}

El estudio se realizó según el enfoque cuantitativo, pues las variables se midieron con base en una escala numérica y los datos se analizaron utilizando la estadística descriptiva e inferencial. Por su alcance es una investigación descriptiva; se especifican las características importantes de las variables gestión administrativa y conocimiento de las TIC (Hernández et al., 2010). El diseńo fue no experimental, transversal correlacional. Es no experimental porque no se manipularon variables, estas se midieron tal como se manifestaron en la población en el momento en que se administraron los cuestionarios. Es transversal porque la gestión administrativa y el conocimiento de las TIC se midieron en un momento determinado; $y$ correlacional porque los datos se correlacionaron para determinar el grado de relación que hay entre dichas variables (Hernández et al., 2010).

\section{Instrumentos}

El instrumento elegido fue el cuestionario, que permitió recoger información acerca de la percepción que los docentes de la Institución Educativa Innova Schools tienen respecto a las variables gestión administrativa y conocimiento de las TIC.

La validez del instrumento se verificó mediante juicio de expertos. Participaron tres magísteres de la escuela de Post Grado de la Universidad César Vallejo, quienes concluyeron que los instrumentos eran válidos para utilizarse en la investigación, con un promedio de $95 \%$. La confiabilidad se analizó con la base de datos de una administración piloto. En el análisis se empleó el coeficiente de consistencia interna alfa de Cronbach, que dio $\alpha=.85$ para el cuestionario sobre percepción de la gestión administrativa y $\alpha=.67$ para el cuestionario sobre conocimiento de las TIC en los docentes.

\section{Tabla 1}

Descripción del cuestionario variable "Percepción de la gestión administrativa"

\begin{tabular}{|c|c|c|}
\hline Dimensiones & Indicadores & Ítems \\
\hline \multirow{4}{*}{$\begin{array}{l}\text { Gestión de } \\
\text { Recursos } \\
\text { Tecnológicos }\end{array}$} & $\begin{array}{l}\text { Dispone de recursos tecnológicos } \\
\text { en la sala de cómputo. }\end{array}$ & \multirow{4}{*}{$\begin{array}{l}1,2,3 \\
4,5,6 \\
7,8,9 y \\
10\end{array}$} \\
\hline & $\begin{array}{l}\text { Dispone de programas informá- } \\
\text { ticos. }\end{array}$ & \\
\hline & $\begin{array}{l}\text { Dispone de Intranet y canal de } \\
\text { acceso a Internet }\end{array}$ & \\
\hline & $\begin{array}{l}\text { Administración de la sala de } \\
\text { computo }\end{array}$ & \\
\hline \multirow{5}{*}{$\begin{array}{l}\text { Gestión de } \\
\text { Recursos } \\
\text { Financieros }\end{array}$} & $\begin{array}{l}\text { Inversión de la infraestructura de la } \\
\text { sala de cómputo. }\end{array}$ & \multirow{5}{*}{$\begin{array}{l}1, \quad 12, \\
13, \quad 14, \\
15, \quad 16, \\
17, \quad 18, \\
19,20\end{array}$} \\
\hline & $\begin{array}{l}\text { Inversión de adquisición de com- } \\
\text { putadoras }\end{array}$ & \\
\hline & $\begin{array}{l}\text { Inversión de implementación de } \\
\text { redes e internet en la institución. }\end{array}$ & \\
\hline & $\begin{array}{l}\text { Inversión para la adquisición de } \\
\text { plataformas educativas. }\end{array}$ & \\
\hline & $\begin{array}{l}\text { Inversión destinada al mantenimien- } \\
\text { to de recursos tecnológicos de la } \\
\text { institución. }\end{array}$ & \\
\hline
\end{tabular}

Fuente: Elaboración propia. 
Tabla 2

Descripción del cuestionario variable "Conocimiento de las TIC de los docentes del nivel primaria"

\begin{tabular}{|c|c|c|}
\hline Dimensiones & Indicadores & Ítems \\
\hline \multirow{2}{*}{$\begin{array}{l}\text { Conocimiento de } \\
\text { las herramientas } \\
\text { Informáticas }\end{array}$} & $\begin{array}{l}\text { Conoce las herramientas de } \\
\text { ofimática }\end{array}$ & \multirow[t]{2}{*}{$\begin{array}{l}1,2,3,4 \\
5,6,7,8\end{array}$} \\
\hline & Conoce aplicativos informáticos & \\
\hline \multirow[t]{4}{*}{$\begin{array}{l}\text { Conocimiento de } \\
\text { las herramientas } \\
\text { telemáticas }\end{array}$} & $\begin{array}{l}\text { Conoce la herramienta telemática } \\
\text { de internet para mejorar la calidad } \\
\text { de la enseñanza. }\end{array}$ & \multirow{4}{*}{$\begin{array}{l}9, \quad 10 \\
11,12 \\
13,14 y \\
15\end{array}$} \\
\hline & $\begin{array}{l}\text { Conoce la herramienta telemática } \\
\text { de intranet en su práctica } \\
\text { pedagógica }\end{array}$ & \\
\hline & $\begin{array}{l}\text { Conoce la herramienta telemática } \\
\text { de correo electrónico en su práctica } \\
\text { pedagógico. }\end{array}$ & \\
\hline & $\begin{array}{l}\text { Conoce la herramienta telemática } \\
\text { chat para promover el proceso } \\
\text { enseñanza-aprendizaje. }\end{array}$ & \\
\hline \multirow[t]{5}{*}{$\begin{array}{l}\text { Conocimiento de } \\
\text { Internet }\end{array}$} & $\begin{array}{l}\text { Conoce la herramienta telemática } \\
\text { skype como apoyo para la } \\
\text { enseñanza. }\end{array}$ & \multirow[t]{5}{*}{$\begin{array}{l}16,17 \\
18,19 \\
20,21\end{array}$} \\
\hline & $\begin{array}{l}\text { Conoce la herramienta telemática } \\
\text { de plataforma educativa como } \\
\text { apoyo para la enseñanza. }\end{array}$ & \\
\hline & $\begin{array}{l}\text { Conoce los portales de páginas web } \\
\text { como apoyo para la enseñanza. }\end{array}$ & \\
\hline & $\begin{array}{l}\text { Conoce software online para } \\
\text { promover el proceso enseñanza- } \\
\text { aprendizaje }\end{array}$ & \\
\hline & $\begin{array}{l}\text { Conoce la herramienta telemática } \\
\text { de redes sociales para promover el } \\
\text { proceso enseñanza-aprendizaje. }\end{array}$ & \\
\hline
\end{tabular}

Fuente: Elaboración propia.

\section{Procedimiento}

El trabajo se desarrolló en cuatro etapas bien definidas:

Primera etapa: Búsqueda de información bibliográfica y antecedentes. Consistió en explorar la literatura acerca de las variables gestión administrativa y conocimiento de las TIC. La búsqueda se hizo en importantes bases de datos disponibles en las páginas web de distintas organizaciones avocadas a la difusión de información y conocimiento especializado de distintas áreas.

Segunda etapa: Elaboración de materiales. Una vez acopiada la información teórica, se diseñó el cuestionario que permitiría recolectar la información acerca de las variables (gestión administrativa y conocimiento de las TIC) cuya correlación se debía verificar. Dicho instrumento fue sometido a verificación de su validez y confiabilidad para utilizarse en la investigación.

Tercera etapa: Investigación de Campo. Comprobada la validez del cuestionario y el grado de confiabilidad de los datos que proporcionaba, se procedió a administrarlo en tiempo único a la muestra, pues el estudio es de naturaleza no experimental, transversal correlacional.

\section{Cuarta etapa: Sistematización de análisis e in-} terpretación de los resultados. . Los datos se sistematizaron en una matriz Excel y posteriormente en el software estadístico SPSS v.21. Se comprobó que la base de datos no presentaba error que pudiera afectar los resultados y a continuación se realizó el análisis descriptivo e inferencial usando los programas ya mencionados. Los resultados se presentaron mediante tablas y gráficos estadísticos, teniendo como referencia la media, desviación estándar. Los puntajes mínimo y máximo y las frecuencias porcentuales.

\section{Resultados}

El análisis inferencial se realizó considerando ,05 de nivel de significancia ( $5 \%$ de error). La decisión se adoptó teniendo en cuenta si el $p$-valor de la prueba es menor o mayor que, 05 . Es decir;

$\mathrm{Si} p>.05$, no se rechaza la $\mathrm{H}_{0}$. $\mathrm{Si} p<.05$, se rechaza la $\mathrm{H}_{0}$ y se concluye con $\mathrm{H}_{\mathrm{a}}$.

Los resultados de la investigación (tabla 3) demostraron que el puntaje promedio en la percepción de la gestión administrativa fue 86 puntos con una variación de 8 puntos; además, los puntajes oscilaron entre 56 y 98 puntos. En cuanto al Conocimiento de las TIC, el puntaje promedio alcanzado por los docentes fue 81, con una variación de 7 puntos, con valores que oscilaron entre 64 y 94 puntos. 
Gestión administrativa y conocimiento de las TIC en docentes de educación primaria de las instituciones educativas Innova Schools de San Juan de Lurigancho y Ate

Tabla 3

Puntajes directos de la gestión administrativa y el conocimiento de las TIC en docentes del nivel Primaria de la Institución Educativa "Innova Schools", de los distritos de San Juan de Lurigancho y Ate.

\begin{tabular}{lcccc}
\hline $\begin{array}{l}\text { Variablel } \\
\text { Dimensiones }\end{array}$ & Media & $\begin{array}{l}\text { Desviación } \\
\text { Estándar }\end{array}$ & Mín. & Máx. \\
\hline $\begin{array}{l}\text { Gestión administrativa } \\
\text { - Gestión de Recursos }\end{array}$ & 86 & 8 & 56 & 98 \\
$\quad \begin{array}{l}\text { Tecnológicos } \\
\text { Gestión de Recursos }\end{array}$ & 42 & 4 & 28 & 50 \\
$\quad$ Financieros & 81 & 7 & 64 & 94 \\
\hline $\begin{array}{l}\text { Percepción del Conocimiento } \\
\text { de las TIC de los docentes del } \\
\text { nivel Primaria }\end{array}$ & & & & \\
\hline
\end{tabular}

Fuente: Cuestionarios sobre gestión administrativa y conocimiento de las TIC (2013).

El análisis descriptivo de los resultados de las variables se muestra en la tabla 4 . Se aprecia que $56 \%$ de docentes perciben que la gestión administrativa es regular, el $40 \%$ de docentes perciben que la gestión de recursos tecnológicos es regular y el $42 \%$ de docentes perciben que gestión de recursos financieros es buena (tabla 4).

\section{Tabla 4}

Niveles de la gestión administrativa y sus dimensiones en la Institución Educativa "Innova Schools", en los distritos de San Juan de Lurigancho y Ate.

\begin{tabular}{|c|c|c|c|c|c|c|c|c|}
\hline \multirow{3}{*}{$\begin{array}{l}\text { Variables/ } \\
\text { Dimensiones }\end{array}$} & \multicolumn{6}{|c|}{ Niveles } & & \\
\hline & \multicolumn{2}{|c|}{ Mala } & \multicolumn{2}{|c|}{ Regular } & \multicolumn{2}{|c|}{ Buena } & \multicolumn{2}{|c|}{ Total } \\
\hline & $\mathrm{N}^{\circ}$ & $\%$ & $\mathrm{~N}^{\circ}$ & $\%$ & $\mathrm{~N}^{\circ}$ & $\%$ & $\mathrm{~N}^{\circ}$ & $\%$ \\
\hline $\begin{array}{l}\text { Gestión } \\
\text { administrativa }\end{array}$ & 27 & 17 & 87 & 56 & 41 & 27 & 155 & 100 \\
\hline $\begin{array}{l}\text { - Gestión de } \\
\text { Recursos } \\
\text { Tecnológicos }\end{array}$ & 53 & 34 & 62 & 40 & 40 & 26 & 155 & 100 \\
\hline $\begin{array}{l}\text { - Gestión de } \\
\text { Recursos } \\
\text { Financieros }\end{array}$ & 44 & 29 & 45 & 29 & 66 & 42 & 155 & 100 \\
\hline
\end{tabular}

Fuente: Cuestionarios sobre gestión administrativa y conocimiento de las TIC (2013).

Según la percepción de los docentes, se observa que el $56 \%$ de docentes de la gestión administrativa en la institución educativa Innova Schools" de los distritos de San Juan de Lurigancho y Ate es regular; solo el $27 \%$ de docentes la consideran buena (figura 1).

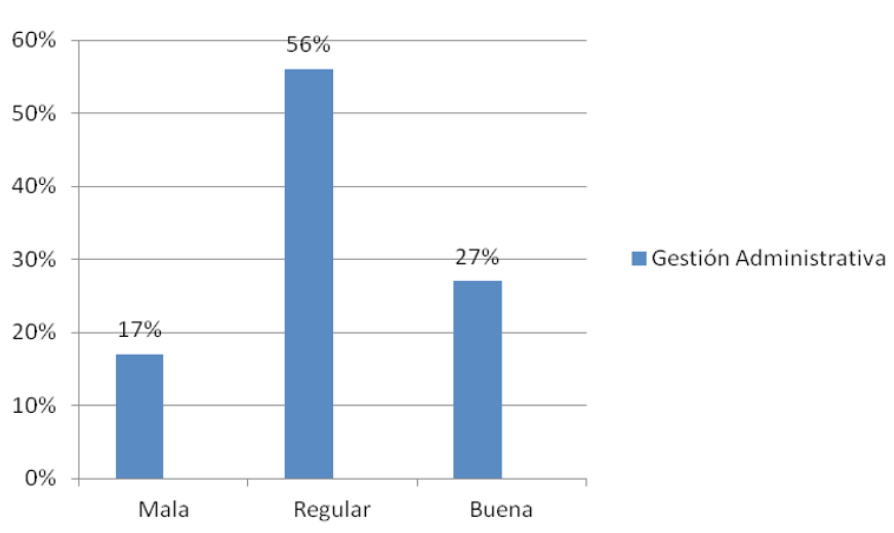

Figura 1. Niveles de la gestión administrativa en la Institución Educativa "Innova Schools" de los distritos de San Juan de Lurigancho y Ate.

Fuente: Cuestionario sobre gestión administrativa (2013).

Según la figura 2, el análisis dimensión por dimensión en la gestión administrativa muestra que el $40 \%$ de docentes la considera regular y solo el $26 \%$ la perciben como buena.

De otro lado, según el $42 \%$ de docentes, la gestión de recursos financieros es buena y para el $29 \%$ regular.

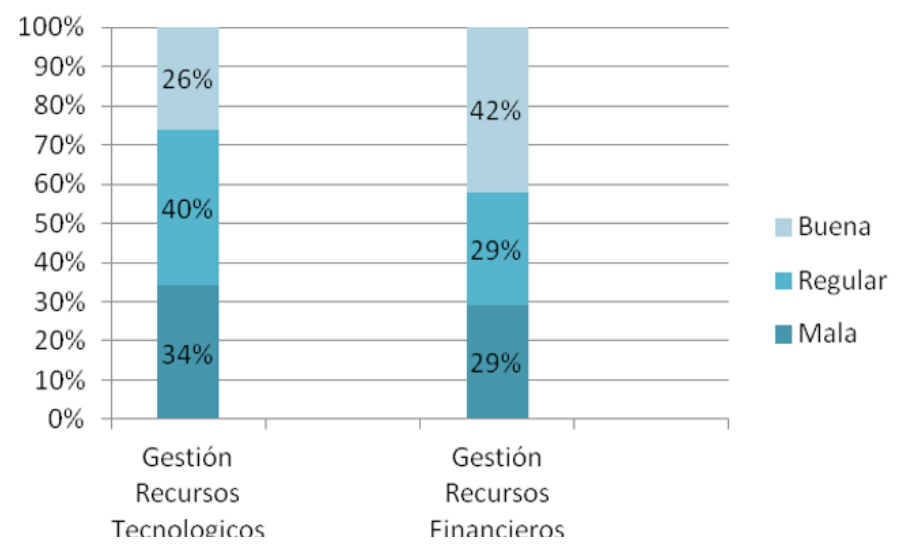

Figura 2. Niveles de las dimensiones de la gestión administrativa en la Institución Educativa "innova Schools" de los distritos San Juan de Lurigancho y Ate. Fuente: Cuestionario sobre gestión administrativa (2013).

Con respecto al análisis del conocimiento de las TIC, en la tabla 5 de la siguiente página se aprecia que el $44 \%$ de docentes la considera de nivel bajo y solo el $24 \%$ de nivel alto. 


\section{Tabla 5}

Niveles de conocimiento de las TIC en docentes del nivel primaria de la Institución Educativa "Innova Schools" de los distritos de San Juan de Lurigancho y Ate

\begin{tabular}{|c|c|c|c|c|c|c|c|c|}
\hline \multirow[b]{3}{*}{ Variable } & \multicolumn{6}{|c|}{ Niveles } & & \\
\hline & \multicolumn{2}{|c|}{ Mala } & \multicolumn{2}{|c|}{ Regular } & \multicolumn{2}{|c|}{ Buena } & \multicolumn{2}{|c|}{ Total } \\
\hline & N. ${ }^{\circ}$ & $\%$ & N. ${ }^{\circ}$ & $\%$ & N. ${ }^{\circ}$ & $\%$ & N. ${ }^{\circ}$ & $\%$ \\
\hline $\begin{array}{l}\text { Percepción del } \\
\text { Conocimiento } \\
\text { de las TIC en } \\
\text { docentes del } \\
\text { nivel primaria }\end{array}$ & 68 & 44 & 49 & 32 & 38 & 24 & 155 & 100 \\
\hline
\end{tabular}

Fuente: Cuestionario sobre conocimiento de las TIC (2013).

En la tabla 6, que asocia la gestión administrativa y el conocimiento de las TIC en docentes del nivel primaria, se observa que para el $67.3 \%$ de docentes hay asociación entre la gestión administrativa de nivel regular y el conocimiento de las TIC del nivel medio. Asimismo, para el 52,9\% de docentes hay asociación entre la gestión administrativa de nivel regular y el conocimiento de las TIC del nivel bajo. Finalmente, el $47.4 \%$ de docentes coinciden en que hay asociación entre la gestión administrativa regular y la percepción del conocimiento de las TIC del nivel alto.

\section{Tabla 6}

Relación entre la gestión administrativa y el conocimiento de las TIC en la Institución Educativa "Innova Schools" de los distritos de San Juan de Lurigancho y Ate

\begin{tabular}{|c|c|c|c|c|c|c|c|c|}
\hline \multirow{3}{*}{$\begin{array}{l}\text { Gestión } \\
\text { Administrativa }\end{array}$} & \multicolumn{6}{|c|}{$\begin{array}{l}\text { Percepción del conocimiento de las } \\
\text { TIC en el nivel primaria }\end{array}$} & & \\
\hline & \multicolumn{2}{|c|}{ Mala } & \multicolumn{2}{|c|}{ Regular } & \multicolumn{2}{|c|}{ Buena } & \multicolumn{2}{|c|}{ Total } \\
\hline & N. ${ }^{\circ}$ & $\%$ & N. ${ }^{\circ}$ & $\%$ & N. ${ }^{\circ}$ & $\%$ & N. ${ }^{\circ}$ & $\%$ \\
\hline Mala & 19 & 27,9 & 5 & 10,2 & 3 & 7,9 & 27 & 17,4 \\
\hline Regular & 36 & 52,9 & 33 & 67,3 & 18 & 47,4 & 87 & 56,1 \\
\hline Buena & 13 & 19,1 & 11 & 22,4 & 17 & 44,7 & 41 & 26,5 \\
\hline Total N & 68 & 44 & 49 & 31 & 38 & 25 & 155 & 100 \\
\hline
\end{tabular}

Fuente: Cuestionario sobre gestión administrativa (2013).

Hipótesis general.

$\mathrm{H}_{0}=$ No existe relación directa y significativa entre la gestión administrativa y el conocimiento de las TIC, según la percepción de los docentes del nivel primaria de la Institución Educativa Innova Schools en las sedes de San Juan de Lurigancho y
Ate en el año 2013.

$\mathrm{H}_{\mathrm{a}}=$ Existe relación directa y significativa entre la gestión administrativa y el conocimiento de las TIC, según la percepción de los docentes del nivel primaria de la Institución Educativa Innova Schools en las Sedes de San Juan de Lurigancho y Ate en el año 2013.

El contraste de hipótesis mediante el coeficiente de correlación rho de Spearman dio $\rho=.335^{* *}$, indicando una correlación baja, pero significativa $(p<.01)$ entre las variables gestión administrativa y conocimiento de las TIC (tabla 7).

\section{Tabla 7}

Resultado de la correlación entre las variables gestión administrativa y conocimiento de las TIC en la Institución Educativa Innova Schools, sedes de San Juan de Lurigancho y Ate, 2013

\begin{tabular}{lcc}
\hline \multirow{2}{*}{$\begin{array}{l}\text { Variable: } \\
\text { Gestión administrativa }\end{array}$} & \multicolumn{2}{c}{$\begin{array}{c}\text { Prueba de Correlación } \\
\text { de Spearman }\end{array}$} \\
\cline { 2 - 3 } & $\rho$ & p-valor \\
\hline Conocimiento de las TIC & $0.335^{\star *}$ & 0.00
\end{tabular}

Fuente: Cuestionarios sobre gestión administrativa y conocimiento de las TIC (2013).

Decisión. Considerando que la prueba rho de Spearman dio $\rho<.01$ para la relación entre las variables gestión administrativa y conocimiento de las TIC, al .000 de error se rechaza la hipótesis nula y se concluye que existe relación directa y significativa entre la gestión administrativa y el conocimiento de las TIC, según la percepción de los docentes del nivel primaria de la Institución mencionada.

En la figura 3 de la página siguiente se observa la dispersión de los datos, que determinó la baja correlación entre las variables baja gestión administrativa y conocimiento de las TIC. 
Gestión administrativa y conocimiento de las TIC en docentes de educación primaria de las instituciones educativas Innova Schools de San Juan de Lurigancho y Ate

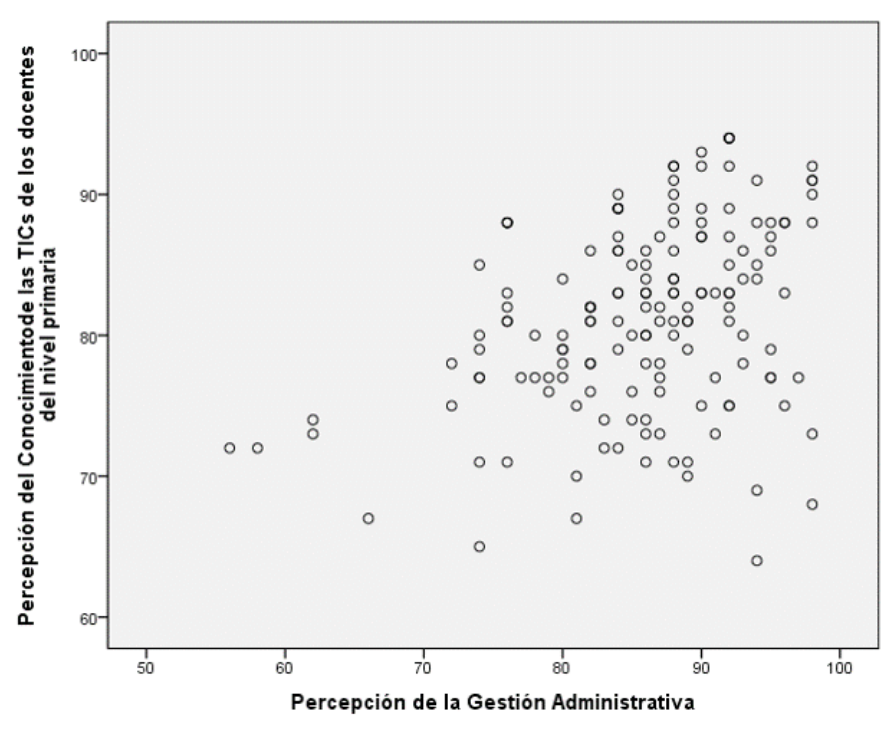

Figura 3. Diagrama de dispersión para la correlación entre la gestión administrativa y el conocimiento de las TIC del nivel primaria.

Fuente: Cuestionarios sobre gestión administrativa y conocimiento de las TIC (2013).

\section{Hipótesis Específica 1}

$\mathrm{H}_{0}=$ No existe relación directa y significativa entre la gestión de recursos tecnológicos y conocimiento de las TIC, según la percepción de los docentes del nivel primaria de la Institución Educativa Innova Schools en las Sedes de San Juan de Lurigancho y Ate.

$\mathrm{H}_{\mathrm{a}}=$ Existe relación directa y significativa entre la gestión de recursos tecnológicos y conocimiento de las TIC, según la percepción de los docentes del nivel primaria de la Institución Educativa Innova Schools en las Sedes de San Juan de Lurigancho y Ate.

El contraste de hipótesis mediante el coeficiente de correlación rho de Spearman dio $\rho=.177^{* *}$, indicando una correlación muy baja, pero significativa $(p<.01)$ entre la gestión de recursos tecnológicos de la variable gestión administrativa y el conocimiento de las TIC en la Institución Educativa "Innova Schools", sedes de San Juan de Lurigancho y Ate (tabla 8).
Tabla 8

Resultado de la correlación entre la gestión de recursos tecnológicos y el conocimiento de las TIC

\begin{tabular}{lcc}
\hline Variable: & \multicolumn{2}{c}{$\begin{array}{c}\text { Prueba de Correlación } \\
\text { Gestión de recursos }\end{array}$} \\
\cline { 2 - 3 } $\begin{array}{l}\text { tecnológicos de la variable } \\
\text { gestión administrativa }\end{array}$ & $\rho$ & p-valor \\
\hline Conocimiento de las TIC & $0.177^{\star *}$ & 0.00
\end{tabular}

Fuente: Cuestionarios sobre gestión administrativa y conocimiento de las TIC (2013).

Decisión. Como la prueba $\rho$ de Spearman dio $p$ $<.01$ para la relación entre la gestión de recurso tecnológicos y el conocimiento de las TIC, al .000 de error se rechaza la hipótesis nula y se concluye que existe relación directa y significativa entre la gestión de recursos tecnológicos y conocimiento de las TIC, según la percepción de los docentes del nivel primaria. En la figura 4 se observa la dispersión de los datos de la dimensión gestión de recursos tecnológicos de la gestión administrativa y la variable conocimiento de las TIC, que determinó la correlación muy baja, pero significativa entre estas variables.

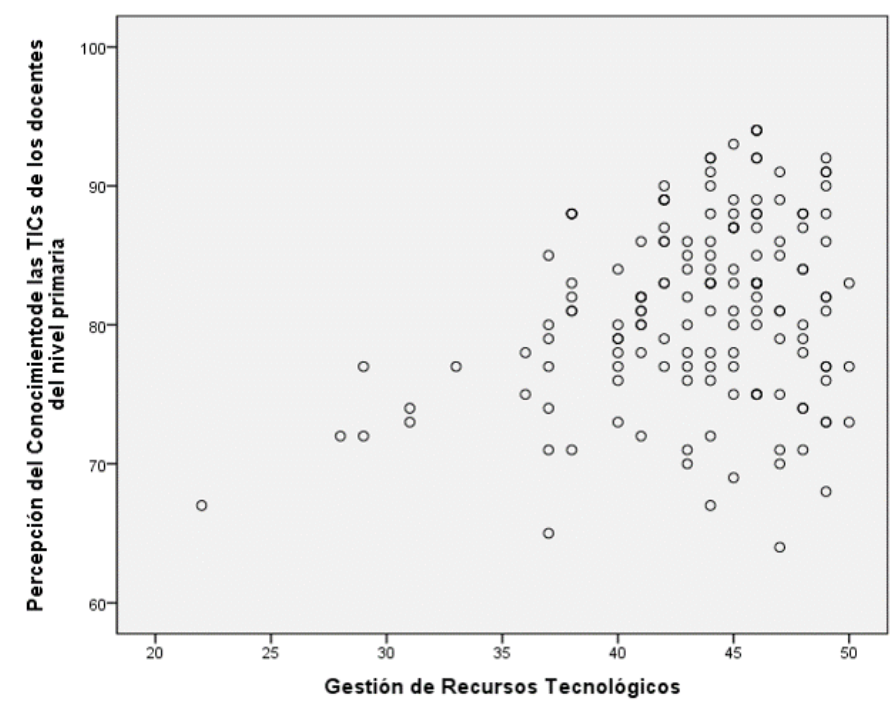

Figura 4. Diagrama de dispersión para la correlación entre la gestión de recursos tecnológicos de la gestión administrativa y el conocimiento de las TIC.

Fuente: Cuestionarios sobre gestión administrativa y conocimiento de las TIC (2013).

\section{Hipótesis Específica 2}

$\mathrm{H}_{0}=$ No existe relación directa y significativa entre la gestión de recursos financieros y conocimiento de las TIC, según la percepción de los docentes del nivel primaria. 
$\mathrm{H}_{\mathrm{a}}=$ Existe relación directa y significativa entre la gestión de recursos financieros y conocimiento de las TIC, según la percepción de los docentes del nivel primaria.

El contraste de hipótesis mediante el coeficiente de correlación $\rho$ de Spearman dio rho $=.368^{* *}$, indicando una correlación muy baja, pero significativa $(p<.01)$ entre la gestión de recursos financieros de la variable gestión administrativa y el conocimiento de las TIC (tabla 9).

\section{Tabla 9}

Resultado de la correlación entre la gestión de recursos financieros y el conocimiento de las TIC

\begin{tabular}{lcc}
\hline Variable: & \multicolumn{2}{c}{$\begin{array}{c}\text { Prueba de Correlación } \\
\text { Gestión de recursos }\end{array}$} \\
\cline { 2 - 3 } financieros de la variable & \multicolumn{2}{c}{ dearman } \\
gestión administrativa & $\rho$ & p-valor \\
\hline Conocimiento de las TIC & $0.368^{* *}$ & 0.00
\end{tabular}

Fuente: Cuestionarios sobre gestión administrativa y conocimiento de las TIC (2013).

En la figura 5 se observa la dispersión de los datos la dimensión gestión de recursos financieros de la gestión administrativa y la variable y conocimiento de las TIC, que determinó la correlación baja, pero significativa correlación entre estas variables.

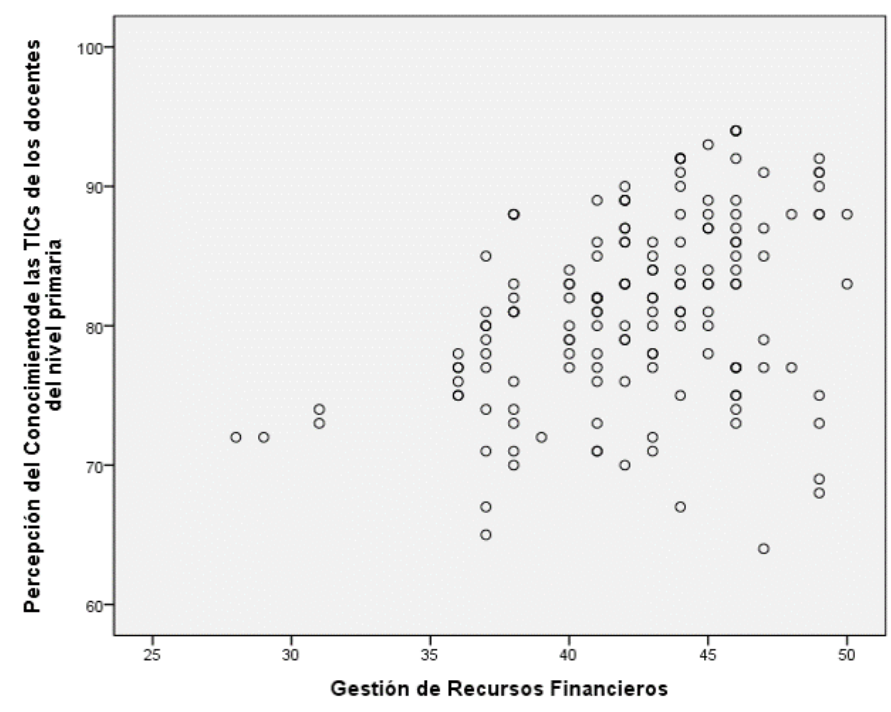

Figura 5. Diagrama de dispersión para la correlación entre la gestión de recursos financieros de la gestión administrativa y el conocimiento de las TIC en la institución educativa "Innova Schools" de los distritos San Juan de Lurigancho y Ate.

Fuente: Cuestionarios sobre gestión administrativa y conocimiento de las TIC (2013).

\section{DisCUSIÓN Y CONCLUSIONES}

En primer lugar se buscó determinar el nivel predominante en cada una de las variables de estudio $y$, en segundo lugar, detectar la relación que existe en cada una de las dimensiones de la gestión administrativa y la percepción del conocimiento de las TIC, según percepción de los docentes del nivel primaria. Los resultados de la prueba de hipótesis general indicaron que entre la gestión administrativa y el conocimiento de las TIC existe una relación baja $\left(\rho=.335^{* *}\right)$, pero significativa $(p<$ $.01)$. Esta percepción de los docentes se relaciona con las comprobaciones realizadas por Mejía (2011), al concluir que las TIC son herramientas que ayudan a mejorar los procesos de enseñanza y aprendizaje, a pesar que en los últimos años los docentes las utilizan dejando de lado la importancia pedagógica que estas deberían tener. Por su parte Martínez y Giraldo (2012) en su estudio sobre la organización y su adaptación a las TIC en los procesos de gestión, concluyeron que estas herramientas ayudan en la toma de decisiones, en la gestión de conocimiento y en el proceso de adaptación de la organización al entorno en que brinda sus servicios. Farjart (2009) concluye que la percepción de los profesores es favorable a las TIC y su actitud hacia el uso de las herramientas informáticas es positiva, por lo que recomiendan ampliar su uso; sin embargo, es necesario capacitarlos para que las usen no solo como un vínculo de comunicación, transmisión de materiales o de asignación de tareas, sino para hacer más interactiva una asignatura aprovechando que los estudiantes se están familiarizando cada vez más con las TIC fuera de los salones de clase. El uso de las TIC no puede ser una moda que hay que seguir en la docencia, utilizando herramientas sin saber siquiera para qué sirven, sino que deben ser escogidas con juicios críticos para que realmente ayuden a mejorar la calidad del proceso educativo. Como lo refiere Selwood (s.f) en su estudio al encontrar que los docentes en un $71 \%$ indicaron que las TIC pueden hacer más productiva su labor pedagógica, el 46\% índico que tiene un mejor desempeño en sus funciones de gestión al trabajar con las TIC y el 53\% que las TIC son utilizadas eficazmente por su escuela para administrar los recursos. 
Gestión administrativa y conocimiento de las TIC en docentes de educación primaria de las instituciones educativas Innova Schools de San Juan de Lurigancho y Ate

Los resultados del contraste de hipótesis para determinar la relación entre la gestión de recursos tecnológicos y el conocimiento de las TIC, según la percepción de los docentes; indicaron que existe una relación muy baja (rho $=.177)$, pero significativa $(\mathrm{p}<.01)$ entre la gestión tecnológica y el conocimiento de las TIC. Dentro de la gestión administrativa, la gestión de los recursos tecnológicos es importante. Resultados que coinciden con Joo (2004) al concluir en su investigación que en el área administrativa de la educación, las TIC sirven para agilizar los procesos administrativos y se han convertido en soporte de la actividad docente. La gestión de recursos tecnológicos no solo debe favorecer la administración educativa, sino también la gestión del aprendizaje en el aula. Oyedemi, (2015) indica que las herramientas tecnológicas en la escuela son positivas por aquellas personas que están a cargo de la administración y gestión de esta, al permitir solucionar problemas de comunicación, lograr una mejor planificación y gestión en las tareas administrativas en las áreas curricular, de relación escuela-comunidad educativa, procesamiento de la información del personal docente, matrículas y otras actividades inherentes a su función, en cuanto al aspecto pedagógico, la calidad del proceso de enseñanza aprendizaje, utilizar nuevos estrategias didácticas mediada por las TIC.

Los resultados de la prueba de hipótesis para determinar la relación entre la gestión de recursos financieros y el conocimiento de las TIC, según la percepción de los docentes, indicaron que existe una relación baja $\left(\rho=.368^{* *}\right)$, pero significativa $(\mathrm{p}<.01)$ entre la gestión financiera y el conocimiento de las TIC. La gestión de recursos debe orientarse a la oportuna y adecuada implementación de tecnología en las escuelas, pensando en que esta favorece los aprendizajes de los estudiantes. Deben tenerse en cuenta las recomendaciones de Fajart (2009) usar las TIC en el aprendizaje, pues mejoran el aprendizaje. En consecuencia, una parte importante de los recursos financieros deben gestionarse hacia la adquisición de nuevas tecnologías de la información y comunicación.

A pesar de los cambios incesantes que ha tenido la tecnología, aún persisten líneas que no están siendo investigadas a la par de estos cambios, como es el caso de este estudio, viéndose reflejado en trabajos no tan actualizados, lo cual ha permitido hacer una reflexión, que a pesar de tener al alcance de sus posibilidades herramientas tecnológicas, los docentes todavía tienen una resistencia a utilizar las mismas, como también lo aseveran Oboegbulem \& Ugwu (2013) al mencionar que el uso de las TIC en educación es muy lento, ya que los docentes tienen una falta de experticia en el manejo de las tecnologías, esto sugiere hacer nuevas investigaciones sobre este campo, de porque los docentes están renuentes a utilizar y aplicar las TIC en su vida cotidiana y profesional; así como experimentar y apropiarse de ellas en su quehacer pedagógico y administrativo, quizás haya una relación con aspectos subjetivos y/o emocionales que puedan estar interfiriendo en la aprehensión de estos, sería interesante incluir estas perspectivas psicológicas, aspectos culturales y personales.

\section{REFERENCIAS BIBIOGRÁFICAS}

Adell, J. (1993). Educación en Internet. EE.UU: Universitat Tarraconensis - Departamento de Educación.

Aguerrondo, I. (1990). El planeamiento educativo como instrumento de cambio ( $1^{\mathrm{a}} \mathrm{ed}$.). Buenos Aires: Rayuela Editores Asociados.

Altuna, J. (2010). Integración de Internet en la Didáctica de la asignatura "Conocimiento del Medio" en el Tercer Ciclo de Primaria. Chile: Universidad del País Vasco.

Antúnez, S. (1993). Hacia una gestión autónoma del centro escolar. En: Claves para la organización de centros escolares. Barcelona: ICE/Horsori.

Barbeito, A. (2004). La Gestión de recursos tecnológicos en la escuela: un modelo de capacitación y desarrollo de materiales. Revista de Informática Educativa y Medios Audiovisuales. Buenos Aires: Universidad Tecnológica Nacional, Facultad Regional.

Burbules, N. (2008). Riesgos y promesas de las TIC en la educación. ¿QQué hemos aprendido en estos últimos 10 años? En: Las TIC: del aula a la agenda política. Buenos Aires: IIPE, UNESCO, UNICEF.

Cabero, A. (2007). Nuevas tecnologías aplicadas a la educación. Madrid, Espańa: McGraw-Hill.

Castellanos, O. (2007). Gestión Tecnológica. Bogotá: Facultad de Ingeniería de la Universidad Nacional de Colombia.

Chiavenato, I. (1990). Administración de Recursos Humanos. México: Mc Graw Hill.

Clares, J. (2000). Telemática, enseñanza y ambientes virtuales colaborativos. Revista Comunicar (pp. 191-199), 14 (26). 
Farjart, A. (2009). Percepciones y actitudes de los profesores sobre el uso de las Tecnologías de la Información y Comunicación en el proceso enseńanza-aprendizaje. (Tesis inédita, Universidad Anáhuac Mayab, Mexico). Recuperada de: https://dialnet.unirioja.es/servlet/articulo?codigo=3347260

Gavilán, B. (2008). Guía Básica para la Gestión Económico-Financiera. Recuperada de: http://www.siis.net/es/buscar/Record/153228

Gestión Administrativa (2004). Dirección General de Gestión de Calidad Educativa. Guatemala: Ministerio de educación de Guatemala.

Hernández, R., Fernández, C. y Baptista, P. (2010). Metodología de la Investigación. México: Mac Graw Hill.

Joo, B. (2004). Análisis y propuesta de gestión pedagógica y administrativa de las TICs, para construir espacios que generen conocimiento en el colegio Champagnat. Tesis para optar el grado de Magister en Educación. (Tesis de maestría inédita). Pontifica Universidad Católica del Perú: Lima.

Krishnaveni, R. \& Meenak, J. (2010) Usage of ICT fo information administration in higher education institutions-A study. International journal of environmental science and debelopment, 1 (3), 282-286. Recuperado de: http://www. ijesd.org/papers/55-D461.pdf

Lagos, I. (2011). Percepciones respecto al uso de herramientas TIC en el aula. (Tesis de licenciatura inédita, Universidad Austral de Chile). Recuperada de: http://www.kelluwen.cl/ wp-content/uploads/documentos/tesis/Lagos_Ivonne_Tesis.pdf

Marqués, P. (2008). Les TIC a l'educació social: Entorns de treball i exemples d'ús. Revista Quaderns d'Educació Social (pp. 159-173) .

Martínez, L. (2012). Administración Educativa. México: Red Tercer Milenio S.C.

Martínez, J. y Giraldo, L. (2012). La organización y su adaptación a las tecnologías de la información y la comunicación en procesos de gestión del conocimiento. Semestre Económico, 15 (32), pp. 161-184.

Mejía, N. (2011). Percepciones, Uso y Apropiación de TIC en los Docentes. (Tesis de licenciatura inédita, Facultad de Comunicaciones de la Universidad de Antioquía, Medellín, Colombia). Recuperada de: http://aprendeenlinea.udea. edu.co/lms/investigacion/file.php/60/resultados/percepcion_de_tic_en_docentes_nmh.pdf

Ministerio de Educación. (2009). Diseño Curricular Nacional. Lima: MINEDU.

Morelos, M. (2011). Los recursos tecnológicos en educación: Recursos subutilizados en la actualidad. Revista Digital de Investigación Educativa (pp. 127-144), 2 (7).

Nacho, M. (1999). Introducción a la informática. (2a ed.). Madrid: Anaya Multimedia

Oboegbulem, A. \& Ugwu, R. (2013) The place of ICT (Information and Communication Technology) in the administration of secondary schools in south Eastern States of Nigeria. US-China Education Review, 3 (4), 231-238.
Recuperado de: http://files.eric.ed.gov/fulltext/ED542971. pdf

Oyedemi, O. (2015) ICT and effective school management: Administrators' perspective. Proceedings of the World Congress on engineering, London, U.K. Recuperado de: http:// www.iaeng.org/publication/WCE2015/WCE2015_pp249252.pdf

Pareja, C., Andeyro, A. y Ojeda, M. (1994). Introducción a la Informática. España: Ediciones Pareja.

Perera, F. (2012). Opiniones de los alumnos, docentes y autoridades escolares respecto de las NTIC y su utilización en el proceso de enseñanza aprendizaje. (Tesis de maestría inédita, Tizimín, Yucatán). Recuperada de: http://www. academia.edu/1553789/PERCEPCIONES_DE_LOS_ ALUMNOS_DOCENTES_Y_AUTORIDADES_ESCOLARES_RESPECTO_DE_LAS_NTIC

Reyes, A. (2010). Administración de Empresas. México: Larios.

Romero, H. (1993). Estudio de investigación "Informática Educativa en el Nivel Primario. Universidad Pedagógica Nacional". México: UPN.

Rubio, A. (2010). Base pedagógica de las Herramientas de Comunicación Telemática. Revista Innovación y Experiencias (pp. 1-8), 45(26).

Saavedra, J. (2002). Recursos económicos para la operación de las escuelas: El financiamiento de la educación pública en el Perú. Lima: Grupo de Análisis para el Desarrollo (GRADE)

Salinas, S. (2004). Informática Educativa: Uso y aplicaciones de las Nuevas Tecnologías en el Aula. Madrid: Ideaspropias.

Sánchez, J. (1995). Informática Educativa. Trabajo de investigación. Chile: Departamento de Ciencias de la Computación - Universidad de Chile.

Selwood, I. (s.f) Primary School Teachers'Use of ICT for Administration and Management. Recuperado de: http:// link.springer.com/content/pdf/10.1007\%2Fb104289.pdf

Silva, R., Cruz, E. y Hernández, J. (2013). Sistema de Gestión Digital para mejorar los procesos administrativos de instituciones de Educación Superior: Caso de estudio en la Universidad Autónoma Metropolitana. Perspectiva Educacional, 45 (2), pp. 104-134.

UNESCO. (2011). Manual de Gestión para Directores de Instituciones Educativas. Lima: Lance Gráfica. 